\title{
Performance Analysis of Wireless Mesh Network Using Adaptive Informant Factor Based Optimized Channel Allocation
}

\author{
Chethan $\mathrm{K} \mathrm{C}^{1}$ and T G Basavaraju ${ }^{2}$ \\ ${ }^{1}$ Department of Computer Science Engineering, GEC, Hassan \\ ${ }^{2}$ Department of Computer Science Engineering, Government SKSJTI, Bangalore
}

\begin{abstract}
Wireless mesh network (WMN) has become an important leading technology which provides several types of useful applications such as community network, broadband home network and internet access, etc. The rise in the size of users in WMN has created a degradation of efficiency in a network especially in dense areas due to the clumsy channel allocation and hence creating many challenges for enhancing the users experience, network quality and throughput. Therefore in this paper, we proposed OCA based AIF model that can access the channel information and then it process to improve the RF channel association. The proposed OCA-AIF will function for each period when some interference is detected via AIF and we further extend this analysis by taking in to consideration the influence of interference to provide a high quality indicator in network. The analysis of result shows the optimization by our proposed approach which increases as per the increment of relay nodes (RNs).
\end{abstract}

\section{KEYWORDS}

Wireless Mesh Network (WMN); Adaptive Informant Factor (AIF), Optimized Channel Allocation (OCA), Relay Nodes (RNs)

\section{INTRODUCTION}

In the last several years, there is an extensive demand for portable devices such as tablets and smart phones, which are having user friendly interface, nice affordable price and functionality. These circumstances have created a high demand for wireless spectrum in wireless Mesh networks ("WMNs"). The presence of unutilized licensed or non-licensed frequency spectrum in WMN facing the significant amount of spectrum allocation difficulty, more specially in dense areas such as shopping complex, commercial area etc., where the closer RNs interfaces with each other RNs in order to acquire the spectrum. These types of interferences in between the RNs in a network can cause negative effect in terms of user experiences; also it can degrade the user throughput and provide lower connection quality. The radio signal interference in WMNs has been an advanced topic for the researchers and provide major challenges that has been studied in [1] - [11], also several contribution has adopted in order to build optimized model that tends to tackle the interferences in dynamic channel allocation.

There are different assumptions, made while considering channel allocation for WMNs and hence, the different approaches and solutions are considered by the authors. However, the general 
channel allocation prototype have been studied such as static channel allocation and dynamic channel allocation, where in static allocation the interfaces are allocated channels permanently while in dynamic allocation the interfaces are permitted to switch to another channels. In considering the static channel allocation, the traffic information in network is assumed by the approach due to its collective traffic load at each "mesh-router" deviates infrequently. In [1], they proposed a model to resolve the joint channel assignment and routing issues that can compute a routing approach as well as channel allocation approach, which causes all traffic information to be satisfied. In [2], they used formulation of linear programming with the fairness and interferences constraints. In addition, they assumed an approximation approach to obtain a joint channel assignment and routing scheme.

Moreover, other studies have considered the statistical pattern of traffic in a network instead of appropriate profile of traffic, in [3] they assumed that, the internet gateway traffic from/to the clients is crucial, therefore they initially build a routing tree of load balanced from the actual network topology and afterwards they used a load aware distributed approach to allocate the channels for links on tree. While considering the other scenario, the peer-to-peer traffic was taken to be the essential in [4], where the authors have generated a k-linked backbone from the actual network topology and afterwards they allocated channel on the generated topology in order to minimize the interference. However, a prioritized-MAC (medium access control) convention is proposed for WSNs in [5] by considering the binary count-down methodology. Prioritized-MAC viably disposes the information crash and improves channel designation additionally. It offers strict administration separation for organizing the packets and it can be assessed by impact likelihood, reneging/dropping likelihood and channel effectiveness.

Based on the frequent access to the channels, the dynamic channel allocation can be split into short-term and long-term, where in short-term the channel switching takes place frequently and less frequently in long-term. There are some link level type dynamic allocation strategies has been proposed [6] [7], where the rate of channel switching is very frequent among the nodes and wanted to communicate with the other nodes, therefore it causes the switching overhead and required a coordination mechanism. This dynamic channel allocation approaches can be categorized into different types such as in first one, an individual interface from a node is only purpose to control [6] and this type of model does not need any synchronization among the presented nodes but these may not utilize the resources efficiently. In considering the second type, there will be no separate interface is dedicated to control, therefore the resources may utilize more efficiently but the nodes required synchronization among them [7]. In [8], they showed a different channel allocation scheme for IEEE 802.11, based on systems with point-to-point joins, which are mainly intended for the rural areas. This channel allocation scheme permits consistent full-duplex information exchange on each connection in the system. Therefore, the channel allocation scheme doesn't require any synchronization over the connections as the channel task prevents cross connection interference.

The several approaches for dynamic channel allocation requires very less frequent channel switching which are proposed in [9], [10], [11]. A centralized server monitoring is proposed in [9] for the environmental changes; it re-computes the channel allocation in network and also acknowledge nodes to select channels at a time period. In [10], they shown the channel allocation approach and distributed routing at each flow; it was particularly developed for the network with each nodes maintaining two radios. The different approach of learning has been proposed in [11], that the nodes separately acquire their channel allocation based on the information of channel usage in neighbourhood. In addition to that, the learning approach requires some time to congregate to optimized channel allocation whenever the traffic pattern got changed at each time. Therefore, in this research article we aim to study the link level OCA on dynamic interfaces. 
The rapidly increment of the licensed and the non-licenced users in WMN has created a challenge for the user experience, network quality and throughput, which causes degradation of efficiency in a network notably in a dense areas due to the clumsy channel allocation. In addition, the RNs operational areas are growing significantly; therefore the network architecture availability and virtualization model of network has motivated us to use the full resources present in a network via resource management. In this paper, we are proposing OCA based AIF model that can access the channel information and then it process to improve the RF channel association. The proposed OCA-AIF will function for each period when some interference is detected via AIF and we further extend this analysis for interference influence as the basis, to provide a high quality indicator in network. The organization of RF channels and RNs is done under IEEE 802.11s standard; in result section we compared our proposed OCA-AIF model with respect to other approaches in same environment to validate the performance analysis.

\section{LITERATURE SURVEY}

The WSN is a developing technology that gives high quality service to last customers as the "Last mile" through the internet. The multicast communication is a key for WSNs and among a group of nodes multicast gives efficient data distribution and hence maximize throughput. Multi channel multicast (MCM) approach is proposed to Multichannel Mesh Networks that uses Heuristic channel assignment algorithm to improve the throughput and bandwidth information [12], to provide the efficient routing. A novel route mass is considered to capture all available path and the available path bandwidth and it is used as the maximum additional rate flow which can push before the saturating path. WSNs at high density deployment are very difficult to monitor and its result may cause strong channel interference at dissimilar networks. To address the interference of adjacent channels and co-channels, the researcher uses channel allocation to increase the framework presentation for multiple WSNs. In [13], they proposed an initiative model of a channel interference to compute the interference effect of bit ratio at framework; afterwards the issue is expressed in channel allocation. They also suggested a genetic algorithm for establishment of channel task technique in order to reduce the interference effect.

In [14], they investigated the routing issue and channel allocation for the transfer of information in WMN by considering the half duplex communication. In addition to that, the channel allocation is associated with the interference constraints (here considered linear constraints); which are recognized as a set of "routing constraints". A mixed integer LP (linear programming) is formulated for the issue and suggested to maximize the minimum spare volume link separately. They also showed the impact of sub-issues that originated from the channel allocation and routing process for large size of networks. This type of technique might decrease the issues originated via decreasing the resolving period; also examine the issue for huge networks with the small traffic necessary for the optimal transmission. The capacity reduction in WMNs is somewhere the main object for wireless links amid interference and utilization of mesh router in multiple radio interference with the diverse frequency channel to increase the network capacity. Moreover, another efficient path to utilize a central controller to consign correctness of radio interfaces to frequency channel may significantly decrease the interference; therefore devising a routing protocol involving an interference study is an important feature in order to find a way to get notably throughput gain. Afterwards, the software-defined networking provides extra malleable and wieldy networks through decoupling data plane and control plane, which applied with logically centralized controller in organization and device; generally it should be the appropriate choice. In [15], they provide the theoretical analysis to utilize a regulator in SDN wireless sensor networks to allocate generated channels to make routing decision, and provide static channel assignment model to build a continued interference between the joins.

While considering the multi-channel and multi-radio WMNs, the combined channel task and routing issue has been discovered. In [16], they performed a rate-variable model in order to 
increase the network throughput which uses the physical interference model and it can estimate the capacity of network more accurately. Afterwards, they provided the measured programming model that allows formulating the assignment of combined channel task and issues of integer linear programming. Although achieving 'QoS' provisioning is always an important problem, $\mathrm{CN}$ ('cognitive networks') provide a flexible type construction which allows the efficient sharing of heterogeneous networks with the transmission resources, therefore the secondary networks at the channel switching frequency has a clear impact on the QoS performance at $\mathrm{CN}$; a borrowed channel by a secondary network is return back when demand occur by the primary network side. In [17], initially they proposed a novel secondary channel algorithm and afterwards a flexible CN architecture for IEEE802.11 based WLANs. These proposed CN architecture uses a Geo-location database as the repository of shared channel information, which could be utilized by secondary networks. In addition, the secondary networks claim to exploit the QoS with traffic in secondary networks, so that the features of obtainable channels in the secondary networks and the planned channel allocation algorithm are enhanced by considering the shared spectrum.

In [18], they shown the examined research for using DCNs ('Data centre networks') in wireless network transmission and their works proposes to resolve the congestion issue caused by some "hot nodes" in order to increase the global performance of network. The model of DCN in wireless transmission sees both the adaptive transmission-rate and the wireless interference. However, both material and time completion for task is taken into account and estimated the impact of global performance on the wireless transmission. However, the establishment of this classical prototype express the channel allocation in wireless DCNs as an optimization process and projected a GA (genetic algorithm) based model to address it and the validation is performed to check the efficiency of GA-based algorithm as well as the wireless transmissions in a wireless DCN. In [19], the issue of power control and channel allocation for interference channels with respect to multiple control loops is examined. Here a scheduler assigns the control for transmitting power and the wireless channels based on practical channel situations. This methodology can promise a required QoS and can reduce the regular transmitting power instantaneously. Therefore, the fact is expressed as an optimization issue also it is answered by an older gradient decent technique. It is an offline algorithm suitable for find the optimal allocation rule to set onward. The specified algorithm is complex to apply in the preparation of the channel distribution; hence, a stochastic sub-gradient ascent algorithm is planned; which is established on presenting the practical channel state and it is showed that the stochastic optimal cost joins are little close to the optimal required value in a network.

\section{Proposed Methodology}

Here, we considered the $A$ number of relay nodes $(R N s)$ in WMN which function in radio frequency $(R F)$ channels at standard of IEEE $802.11 \mathrm{~s} / \mathrm{u}$. A relay type network is a wide-ranging network topology that commonly used in WMN, where destination and source are interconnected via some nodes called as RNs. Figure 1 shows the mesh network diagram. In this study, we proposed the OCA-AIF in wireless mesh environment and channel allocation of RNs is based on the AIF. The proposed OCA-AIF will function for each period when some interference is detected via AIF (i.e., specified by threshold value). However, we further extend this analysis to interference influence on the basis to provide a high quality indicator in network, additionally it will integrated with quality and flow based power adjustment to provide optimize resource management. 
International Journal of Computer Science \& Engineering Survey (IJCSES) Vol.9, No.4/5, October 2018

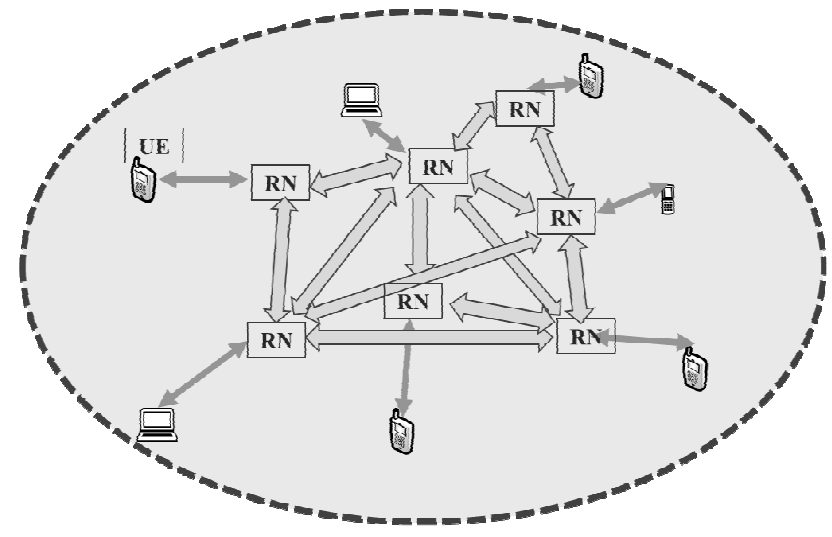

Figure1: Mesh network diagram

The proposed network topology is denoted by $B^{A \times A}$, which is in matrix form and given by;

$B \in\{0,1\}\}^{A \times A}$
$b_{i, j}=\left\{\begin{array}{lr}1, & \text { If exceeds threhold } \\ 0, & \text { Otherwise }\end{array}\right.$

The $R N_{j}$ average power surpasses a specific threshold value, then the value of $b_{i j}$ is set to 1 or else zero, and the $C^{D K A}$ channel allocation matrix is define as;

$C \in\{0,1\}^{D \times A}$

$c_{i, j}=\left[\begin{array}{rr}1, & \text { If } i \text { channel is assigned to } R N_{j} \\ 0, & \text { Otherwise }\end{array}\right.$

The predicted interference matrix at ' $A$ ' RNs is

$F \in F^{A K D}$

However, the available channels are denoted by $D$, the obtained interference level is $E_{i, j}$ in a network where channel $j$ is allocated to $i \mathrm{RN}$. The functional objective because of interference and channel assignment metrics is written as;

$G=H \times C^{T}$

The arrangement of RNs are reflected via $H$, however, the $K$ shows the functional objective in order to represent the obtained interference levels via RNs which are associated due to channel assignment prototype and given as;

$K=G . E$ 
The component matrix multiplication is denotes by ' ' and ' $\mathrm{X}$ ' for matrix multiplication. RN channel selection is process from the interference $E$, therefore, the matrix $\mathrm{E}$ with real-values is presented in (3). While considering real matrix $K$, it can written as $K \in \mathrm{F}^{A \times D}$, where $K$ shows for functional objective that signify the interference magnitude with the RN inter-relation via $H$.

\subsection{Optimized Channel Allocation (OCA)}

The RNs are inconsistence with others RN at channel and given by $H \times C^{T}$, the matrix of OCA is given through $C^{*}$ that deliver minimum level interference in WMN such as;

$c^{*}={ }^{m i n} \sum_{i \leq A} \sum_{j \leq D} k_{i, j}$

The real value of $K$ converted into $\mathrm{dB}$, while the problem associated with OCA has given in equitation (7) and (8)

$$
\begin{aligned}
& C \in\{0.1\}^{D \times A} \text { and, }\|C(:, i)\|_{1}=1 \\
& \forall c_{t, y} \in C: \sum \sum c_{t, y}=A
\end{aligned}
$$

Where, the first norm is $\|.\|_{1}$, which shows the integration of features in each column of $C$.

$$
C^{*}={ }_{C}^{\min } H \times C_{\text {all }}^{T} . E
$$

The constraint given in equation (7) and (8) are assigned to a single channel $\mathrm{RN}$, therefore, $A$ RNs will have total $A$ channels, in order to resolve this problem multi-criteria linear process (MCLP) [20] is considered such as;

$$
\begin{aligned}
& m^{*}={ }_{m}^{\min }\left(n^{T} m\right) \\
& \mu_{m}=p
\end{aligned}
$$

Where, $n$ denotes the coefficient matrix $(n=f(H, E))$, m provide the optimal allocation of channel in the element matrix $C^{*}$. While considering matrix $C^{*}$, the constraint (6) and (7) consist of only non-zero element at every row, however, the matrix $P$ signify the constraint for ' $C$ ' in equation (7) and $m_{i} \in[0,1], \vee i: 1 \leq i \leq A \times D$. Here, the proposed OCA configuration will uphold the minimum interference level in dense WMN, in addition it also important to add complete network scenarios and ones to run MCLP (i.e., from equation (9)). Therefore, the centralized model is used to overcome network status problem through obtaining regular update of RNs. Certain times the RNs cannot able to identify the channels interference, therefore the interference matrix $E$ is used to provide the channel allocation via some network information (i.e., collected through AIF). Therefore, optimal channel configuration is used at open-flow "Network-API" and very much useful in dynamic WMN environment, where the network topology can change sometime. 


\subsection{Large Network Interference Analysis}

As we define that $E$ is interference influences for $A$ RNs and $D$ available channels, where individual $E_{i, d}$ element act as an interference factor and it is integration of all signals corresponding to $d$ channel when it is allocated to $R N_{i}$, it can expressed as;

\begin{tabular}{|l|l|}
\hline$E_{i, d}=\sum_{a \leq A, a \neq i} R_{i, a}(d)=\sum_{a \in A, a \neq i} R_{i}^{t} \beta_{i, a}(d) \varphi_{i, a}(d)$ & (10) \\
\hline
\end{tabular}

Furthermore, the simplified equation can be written as;

$$
E_{i, d}=R_{i}^{t} \sum_{a \leq A, a \pm i} \beta_{i, a}(d) \varphi_{i, a}(d)
$$

Where, $i \geq 1, D \geq d \geq 1, A \geq a$, and $R_{i, a}$ denotes the average power of assigned RF channel and identified the near proximity of $R N_{i}$. The $P_{i}^{t}$ shows the level of transmission power at $R N_{i}$, and the channel gain in between $R N_{i}$ and $R N_{a}$ is denoted by $\beta_{i a}$. While $\varphi_{i a}$ signify the coefficient that varying from 0 to 1 , also the coefficient value will be null/zero for the nonoverlapping channels. Though the estimation and updating should occur in real time scenario, also the actual characteristics of RF-channels should well employ in the network.

The $E$ interference matrix reflects the RNs interference impact in order to improvise the objective of transmission power through considering the orthogonally RF channels, which result in optimized channel allocation $C^{*}$ and minimizes the interference impact throughout the WMN scenario.

In order to check the optimal solution obtained by (8), we have to show the experienced interference with channel assignment via $C^{*}$ in a network, which should be lower than the other channel interference. Here, $E_{I A}$ shows the interference accumulation that can experience at RNs locations and used to represent the large-network quality. The appropriate signal integration obtained at RNs location and created from all the others RNs

$$
E_{l A}=\sum_{i=1}^{A} \sum_{a \leq A, c \pm i}^{A} R_{a}^{t} \beta_{a, i} \varphi_{a, i}
$$

Where, $R_{a,}^{\tau}, \beta_{i, a}$ and $\varphi_{i, a}$ are same as above equation (11), but here we drop $d$ symbol to neglect notation confusion, the resemblance in between $a$ and $i$ value range in (12) with respect to (11) and their recast yields is given as;

$E_{I A}=\sum_{i=1}^{A} \sum_{a \leq A, a \pm i}^{A} R_{a}^{t} \beta_{a, i} \varphi_{a, i}=\sum_{i=1}^{A} \sum_{a \leq A, a \neq i}^{A} R_{\mathrm{c}}^{i} \beta_{i, a} \varphi_{i, a}=\sum_{i=1}^{A} E_{i, d i}$

Where, $d i$ shows for the instance at $d$ that corresponds to $R N_{i}$, therefore the linear integration with coefficients of positive integer of all $E_{i, d}$ is provided to be optimum for $C^{\star}$. To represent any linear summation with the unity coefficient can be given as; 
International Journal of Computer Science \& Engineering Survey (IJCSES) Vol.9, No.4/5, October 2018

\begin{tabular}{|l|l|}
\hline$\sum_{i=1}^{A} E_{i, d} l_{c=c^{*}} \leq\left.\sum_{i=1}^{A} E_{i, d i}\right|_{c=c^{\prime}}$ & (14) \\
\hline
\end{tabular}

Where, $C^{\prime}$ denotes to a particular channel assignment that have at least one different allocated channel from $C^{*}$ and if all the $E_{i, d}$ are positive and larger than the threshold, then applying (13) and (14) we can get;

$$
E_{l A}\left|c-c^{*} \leq E_{l A}\right| c-c^{r}
$$

This denotes the interference throughput status in the network and the obtained information from the RNs, while giving the immediately optimal solution with the $C^{*}$ channel assignment.

\section{RESUlts AND ANALYSIS}

WMNs at high density deployment are very difficult to monitor and its result may cause strong channel interference at dissimilar networks. To address the interference at adjacent channels and co-channels the researchers has to devise an efficient channel allocation to increase the framework presentation for multiple WMNs. Here, we have proposed OCA based AIF model that can access the channel information and then it process to improve the RF channel association. In order to evaluate our proposed approach we used Mat lab 2016b Simulink software for the simulation, with system configuration of Windows 10 operating system, 8 GB RAM, Intel i5 Processor and 2GB Graphics. Here, we have used standard network configuration which is having a "path loss" exponent value, path loss coefficient with the transmit power of $25 \mathrm{dBm}$, the network coverage area of $400 \mathrm{~m} \times 400 \mathrm{~m}$ and 1000 number of total flows is considered for the proposed model evaluation. The objective metric is optimized via AIF, which allow RNs for improved data-rate flow; the AIF based approach is evaluated using OCA to improve the channel assignment process in dense area network. At each time a new flow of downstream will attempt to make a connection, where each novel flow indicates the novel wireless communication by existing user with different requirement 'or' new user with random locality of users. Here, we are considering three scenarios such as scenario-A, scenario-B and scenario-C.

\section{Scenario-A}

In this scenario, we considered $40 \mathrm{RNs}$ at 1000 number of total flows under $400 \mathrm{~m} \times 400 \mathrm{~m}$ network coverage area. The data-rate is computed as per number of flows through different models such as AIF-OCA, AFS [21] and A2OLS [21], data rate is represented in bits-per-second (bps). 
International Journal of Computer Science \& Engineering Survey (IJCSES) Vol.9, No.4/5, October 2018

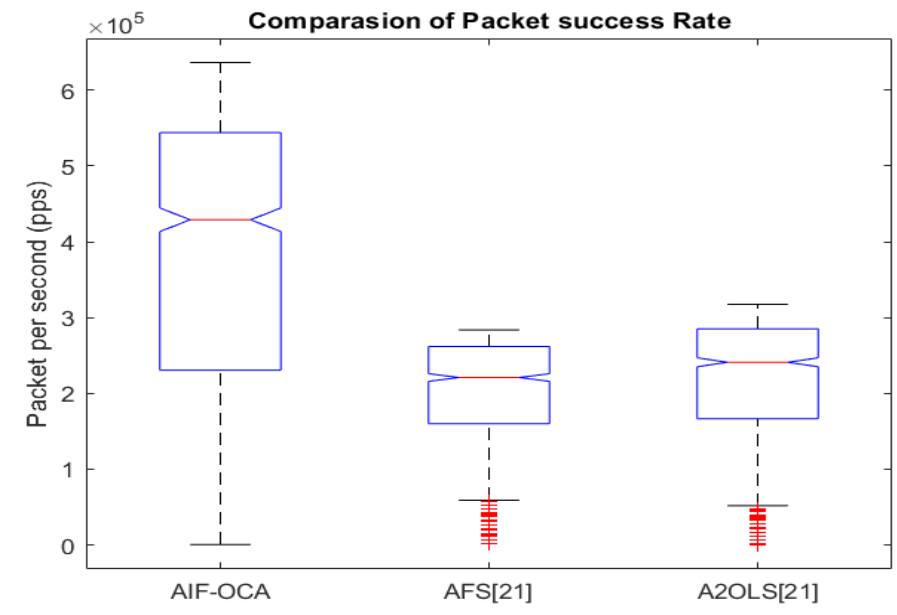

Figure 2: Packet Success Rate ( $p s s)$

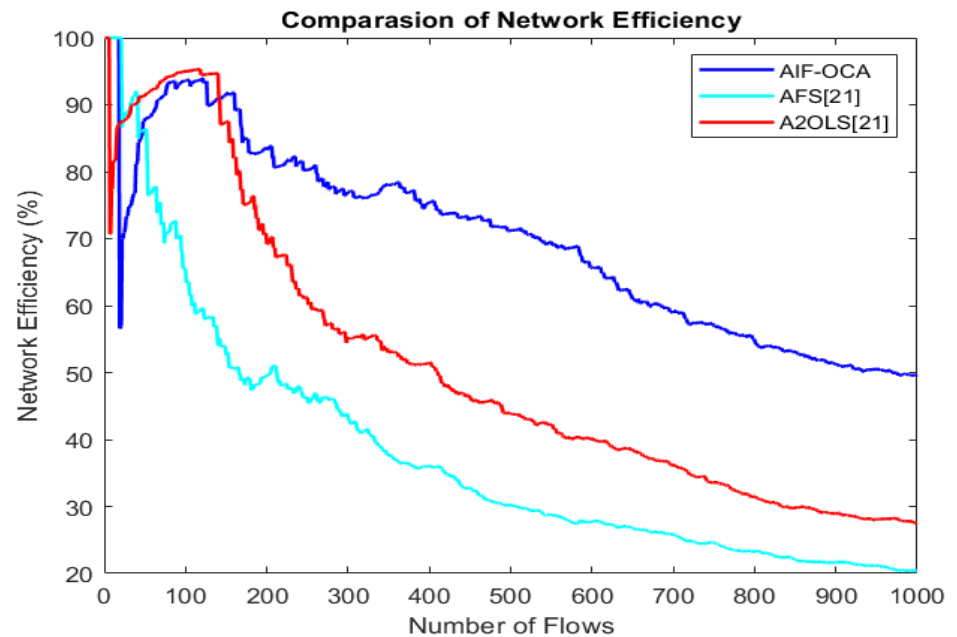

Figure 3: Network Efficiency as per Flows (\%)

Figure 2 shows the packet success rate at different approaches, where the blue box represents the spread of data, red line inside the box represent the mean value packet success rate and small red line outside the box shows the outliners. The packet success rate obtained by AIF-OCA is $0.38 \mathrm{M}$ pps, which is $46 \%$ more compare to AFS [21] and 42\% more compared to A2OLS [21].Figure 3 shows the Network Efficiency as per Flows in percentage, where AIF-OCA network efficiency is $69 \%$, AFS [21] having 37\% and A2OLS [21] having 50\%. 
International Journal of Computer Science \& Engineering Survey (IJCSES) Vol.9, No.4/5, October 2018

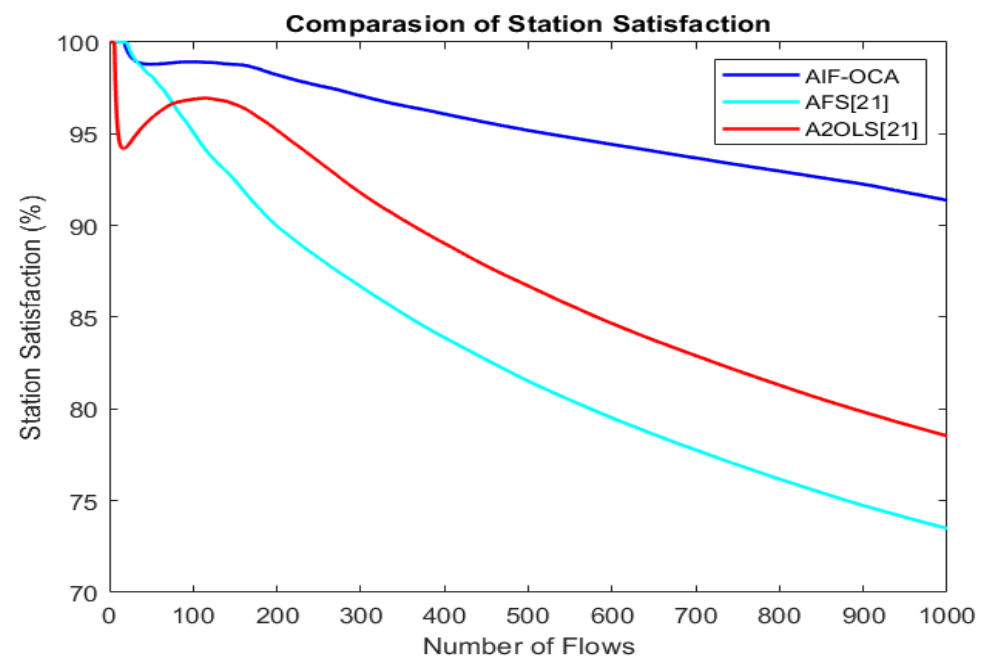

Figure 4: Station Satisfaction (\%)

Figure 4 shows the station satisfaction as per the number of flows, where AIF-OCA obtained is 95\% satisfaction, which is $12 \%$ more compare to AFS [21] and 8\% more w.r.t A2OLS [21].

\section{Scenario-B}

In this scenario, we considered $80 \mathrm{RNs}$ at 1000 number of total flows under $400 \mathrm{~m} \times 400 \mathrm{~m}$ network coverage area. Figure 5 shows the packet success rate at different approaches and each packet can transfer $1 \mathrm{~kb}$ data. The packet success rate obtained by AIF-OCA is $0.43 \mathrm{M}-\mathrm{pps}$, which is $30 \%$ more compared to AFS [21] and 24\% more w.r.t A2OLS [21]. Figure 6 shows the Network Efficiency as per Flows in percentage, where AIF-OCA network efficiency is 79\%, AFS [21] having 51\% and A2OLS [21] having 88\%. Figure 7 shows the station satisfaction as per the number of flows, where AIF-OCA obtained is $96 \%$ satisfaction, which is $7.8 \%$ more compare to AFS [21] and 1.3\% more with respect to A2OLS [21].

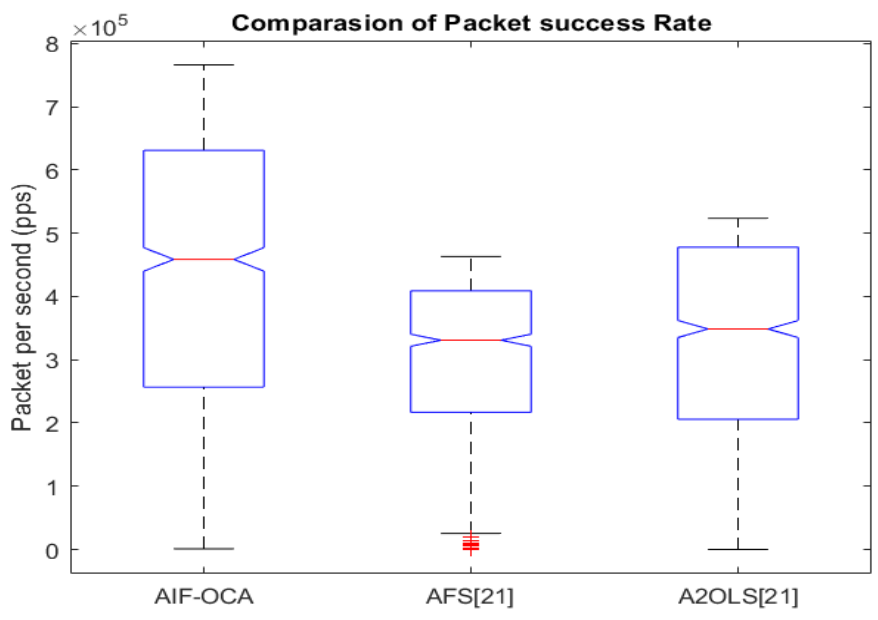

Figure 5: Packet Success Rate (pps) 
International Journal of Computer Science \& Engineering Survey (IJCSES) Vol.9, No.4/5, October 2018

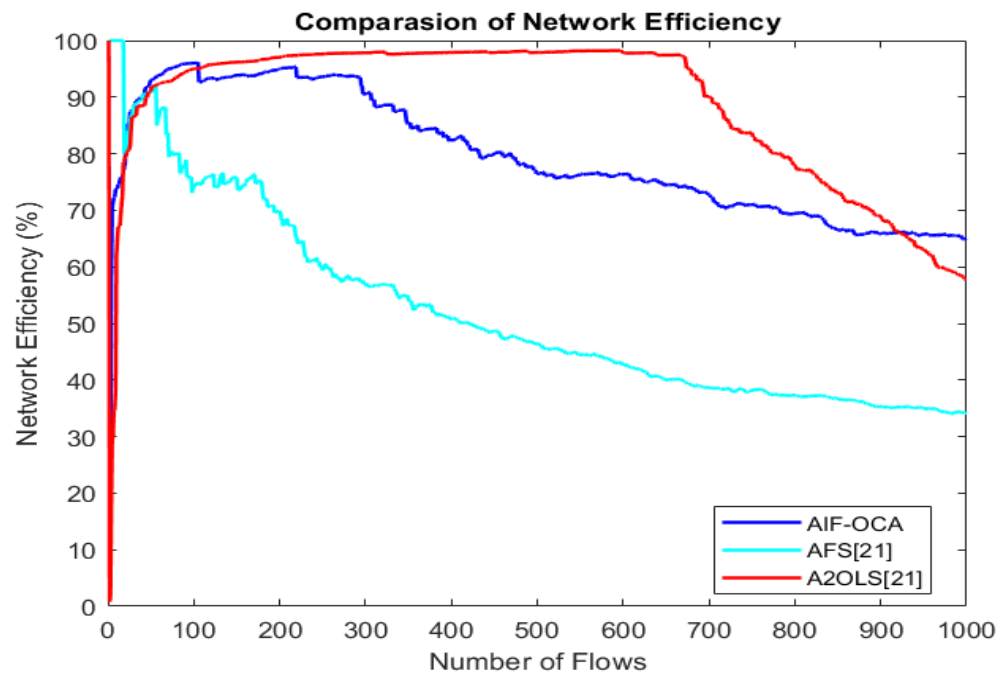

Figure 6: Network Efficiency as per Flows (\%)

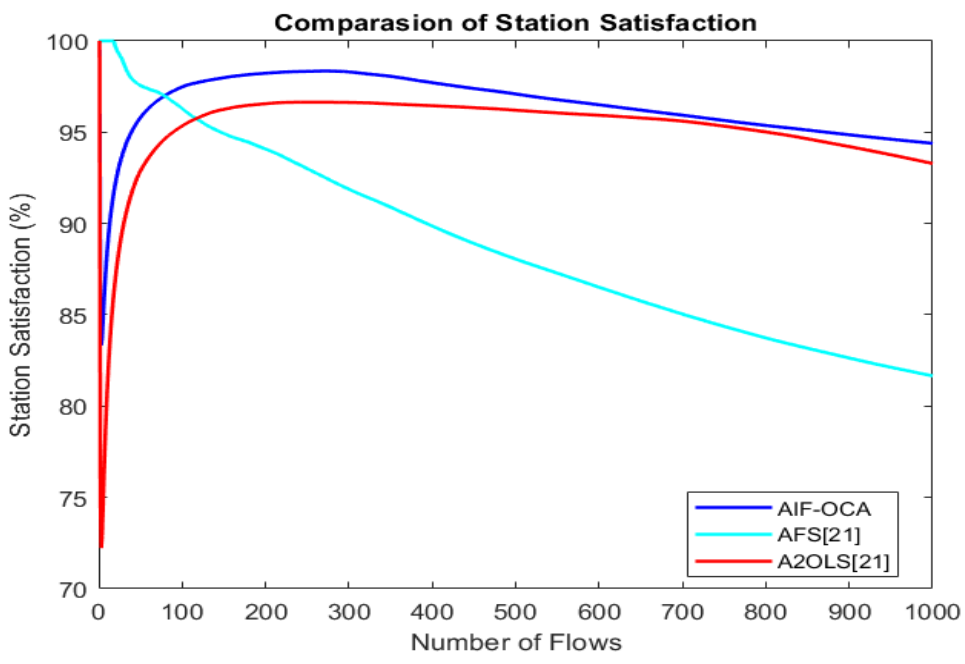

Figure 7: Station Satisfaction (\%)

\section{Scenario-C}

In this scenario, we considered $120 \mathrm{RNs}$ at 1000 number of total flows under $400 \mathrm{~m} \times 400 \mathrm{~m}$ network coverage area. 
International Journal of Computer Science \& Engineering Survey (IJCSES) Vol.9, No.4/5, October 2018

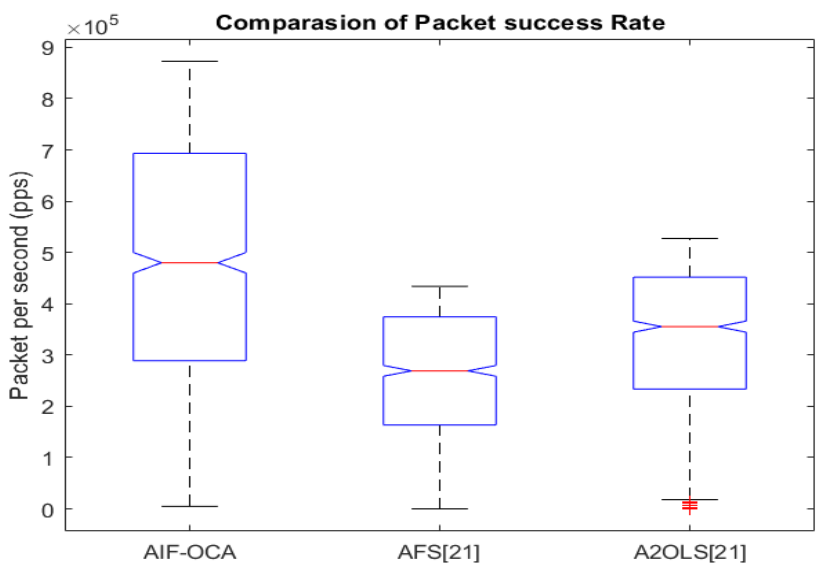

Figure 8: Packet Success Rate ( $p p s$ )

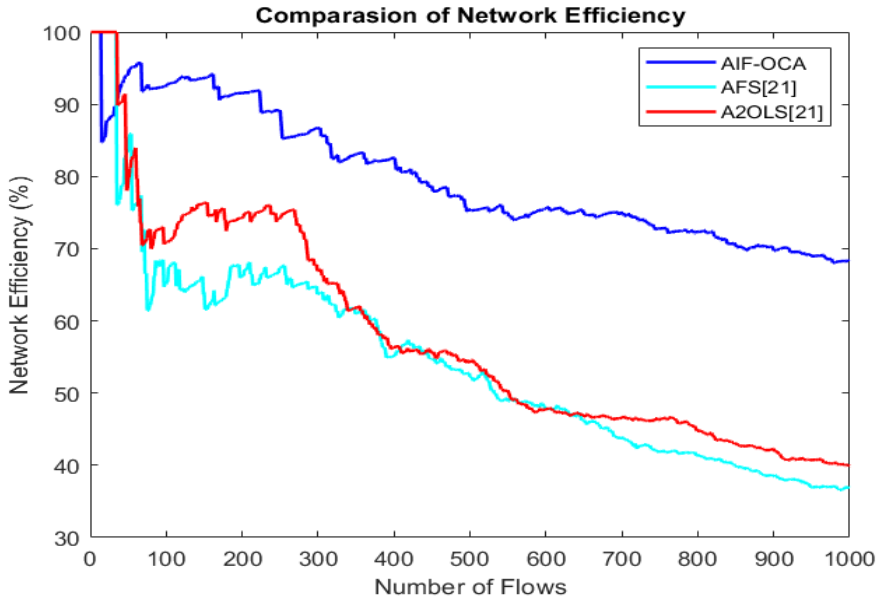

Figure 9: Network Efficiency as per Flows (\%)

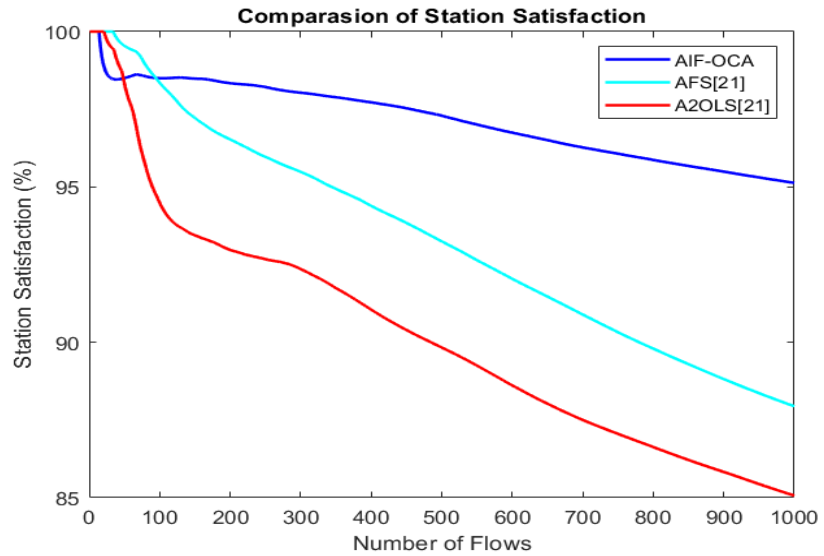

Figure 10: Station Satisfaction (\%) 
Table1: Comparison w.r.t model at different number of nodes

\begin{tabular}{|c|r|r|r|}
\hline $\begin{array}{c}\text { Number } \\
\text { of Nodes }\end{array}$ & AIF-OCA & AFS [21] & $\begin{array}{c}\text { A2OLS } \\
{[21]}\end{array}$ \\
\hline 40 & 571909.9 & 309204.9 & 266055.9 \\
\hline 80 & 714764.5 & 465005.9 & 413014.4 \\
\hline 120 & 847344.2 & 499323.3 & 489546.7 \\
\hline
\end{tabular}

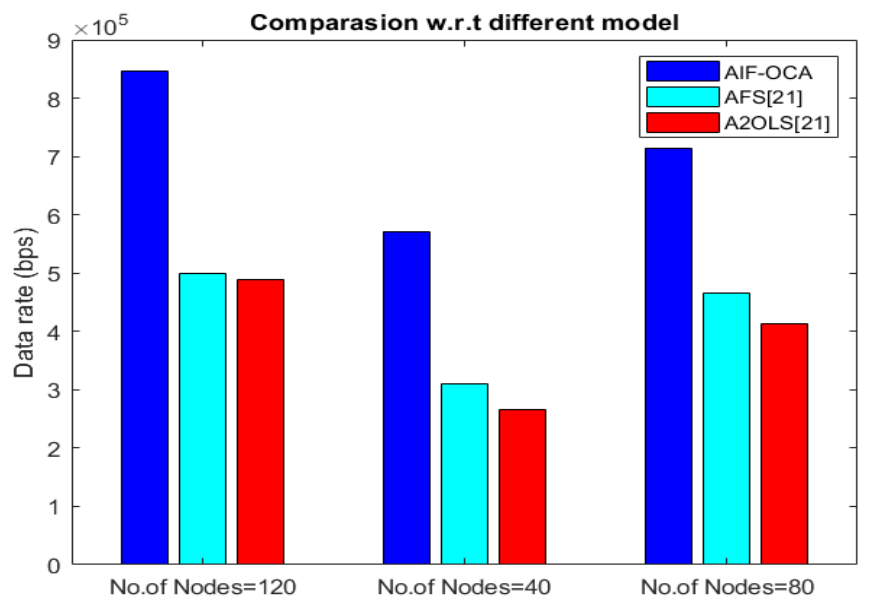

Figure 11: Comparison w.r.t model at different RNs

Figure 8 shows the packet success rate for 1000 flows by considering different approaches, where packet success rate obtained by AIF-OCA is $0.48 \mathrm{M}$-pps, $0.25 \mathrm{M}$-pps by AFS and $0.32 \mathrm{M}$-pps by A2OLS.Our proposed model obtained $47 \%$ more packet success rate compared to AFS [21] and $32 \%$ more w.r.t A2OLS [21]. Figure 9 shows the Network Efficiency as per Flows in percentage, where AIF-OCA network efficiency is 79.8, AFS [21] having 54\% and A2OLS [21] having 57\%. Figure 10 shows the station satisfaction as per the number of flows, where AIF-OCA obtained is 97\% satisfaction, which is 3.8\% more compare to AFS [21] and $7.12 \%$ more with respect to A2OLS [21]. Table 1 shows the average data-rate comparison with respect to model at different number of nodes and their graphical representation is given in figure 11, where at $40 \mathrm{RNs}$ our proposed model performed 45\% more w.r.t AFS and 53\% more w.r.t A2OLS. At 80 RNs, our proposed model performed 34\% more w.r.t AFS and $42 \%$ more w.r.t A2OLS, while considering 120 RNs our proposed model performed $41 \%$ more w.r.t AFS and $42 \%$ more w.r.t A2OLS.

\section{CONCLUSION}

The extensive demand of portable devices has created a high demand of wireless spectrum in WMN. The unutilized licensed or non-licensed frequency spectrum in WMN causes difficulty in channel allocation especially in dense area network, which degrade the users throughput and grants lower connection quality. In this study, we proposed the OCA-AIF in wireless mesh environment and the channel allocation of RNs is based on the AIF. The proposed OCA-AIF will function for each period when some interference is detected via AIF. In result analysis section it has clearly shown that our proposed model got significant amount of improvement w.r.t other existing technique. By increasing the number of RNs improves the data-rate transmission that tends to increase the packet transmission rate. 
International Journal of Computer Science \& Engineering Survey (IJCSES) Vol.9, No.4/5, October 2018

\section{REFERENCES}

[1] A. Raniwala, K. Gopalan, and T. Chiueh,(2004) "Centralized Channel Assignment and Routing Algorithms for Multi-Channel Wireless Mesh Networks," ACM Mobile Computing and Comm. Rev., vol. 8, pp. 50-65.

[2] M. Alicherry, R. Bhatia, and L. Li,(2005) "Joint Channel Assignment and Routing for Throughput Optimization in Multi-Radio Wireless Mesh Networks," Proc. ACM MobiCom.

[3] A. Raniwala and T. Chiueh,(2005) "Architecture and Algorithms for an IEEE 802.11-based MultiChannel Wireless Mesh Network,” Proc. IEEE INFOCOM.

[4] J. Tang, G. Xue, and W. Zhang,(2005)“'Interference-Aware Topology Control and QoS Routing in Multi-Channel Wireless Mesh Networks," Proc. ACM MobiHoc.

[5] L. Pan, H. Wu and N. F. Tzeng,(2009)"An Efficient and Scalable Prioritized MAC Protocol (PMAC) for Backbone Communication in Wireless Sensor Networks," 2009 Third International Conference on Sensor Technologies and Applications, Athens, Glyfada, pp. 508-513.

[6] S.-L. Wu, C.-Y. Lin, Y.-C. Tseng, and J.-P. Sheu,(2000) "A New MultiChannel Mac Protocol with On-Demand Channel Assignment for Multi-Hop Mobile Ad Hoc Networks," Proc. Int'l Symp. Parallel Architectures, Algorithms, and Networks (ISPAN).

[7] J. So and N. Vaidya,(2004)"Multi-Channel Mac for Ad Hoc Networks: Handling Multi-Channel Hidden Terminals Using a Single Transceiver," Proc. ACM MobiHoc.

[8] P. Dutta, S. Jaiswal, D. Panigrahi and R. Rastogi,(2008) "A New Channel Assignment Mechanism for Rural Wireless Mesh Networks," IEEE INFOCOM 2008 - The 27th Conference on Computer Communications, Phoenix, AZ.

[9] K.N. Ramachandran, E.M. Belding, K.C. Almeroth, and M.M. Buddhikot,(2006) "Interference-Aware Channel Assignment in MultiRadio Wireless Mesh Networks,” Proc. IEEE INFOCOM.

[10] S. Pediaditaki, P. Arrieta, and M.K. Marina,(2009) "A Learning-Based Approach for Distributed Multi-Radio Channel Allocation in Wireless Mesh Networks," Proc. IEEE Int'l Conf. Network Protocols (ICNP).

[11] A. Dhananjay, H. Zhang, J. Li, and L. Subramanian,(2009) "Practical, Distributed Channel Assignment and Routing in Dual-Radio Mesh Networks," Proc. SIGCOMM.

[12] D. J. David and V. Jagathesan,(2013) "Efficient channel assignment and routing in wireless mesh network," 2013 International Conference on Current Trends in Engineering and Technology (ICCTET), Coimbatore, 2013, pp. 67-73.

[13] X. Hu, S. Ge and J. Xiao,(2017) "Channel allocation based on genetic algorithm for multiple IEEE 802.15.4-compliant wireless sensor networks," 2017 IEEE International Conference on Signal Processing, Communications and Computing (ICSPCC), Xiamen, pp. 1-5.

[14] S. Kadambar and T. Bodas,(2015) "Joint channel allocation and routing in wireless mesh networks," 2015 Twenty First National Conference on Communications (NCC), Mumbai, 2015, pp. 1-6.

[15] S. Mirzaie and S. Sedaghat,(2017) "Joint channel assignment and routing in Software Defined wireless mesh networks," 2017 Iranian Conference on Electrical Engineering (ICEE), Tehran, 2017, pp. $1515-1519$. 
International Journal of Computer Science \& Engineering Survey (IJCSES) Vol.9, No.4/5, October 2018

[16] C. Yin, R. Yang and W. Zhu, (2016)"Improving channel assignment in wireless mesh network with linear programming," 2016 6th International Conference on Electronics Information and Emergency Communication (ICEIEC), Beijing, pp. 113-116.

[17] S. Gamage, J. Y. Khan and D. T. Ngo,(2016)"A QoS controlled spectrum switching resource allocation technique for cognitive Wi-Fi networks," 2016 IEEE Wireless Communications and Networking Conference, Doha, pp. 1-6.

[18] Y. Cui, H. Wang and X. Cheng,(2011) "Channel allocation in wireless data center networks," 2011 Proceedings IEEE INFOCOM, Shanghai, 2011, pp. 1395-1403.

[19] L. Zhang and J. Sun, (2017)"Channel allocation and power control scheme over interference channels with QoS constraints," 2017 13th IEEE International Conference on Control \& Automation (ICCA), Ohrid, pp. 794-798.

[20] Yong Shi and Heeseok Lee,(1997) "A binary integer linear program with multi-criteria and multiconstraint levels", Computers \& Operations Research, 24, 3, 259 - 273.

[21] L. Wang, K. W. Chin, S. Soh and T. He,(2017) "A Novel Flow-Aware Fair Scheduler for Multi Transmit/Receive Wireless Networks," in IEEE Access, vol. 5, pp. 10456-10468. 